\title{
Casimir force between planes as a boundary finite size effect
}

\author{
Z. Bajnok ${ }^{1}$, L. Palla ${ }^{2}$, and G. Takács ${ }^{1}$ \\ ${ }^{1}$ Theoretical Physics Research Group, Hungarian Academy of Sciences, \\ 1117 Budapest, Pázmány Péter sétány 1/A, Hungary \\ ${ }^{2}$ Institute for Theoretical Physics, Eötvös University, \\ 1117 Budapest, Pázmány Péter sétány 1/A, Hungary
}

\begin{abstract}
The ground state energy of a boundary quantum field theory is derived in planar geometry in $\mathrm{D}+1$ dimensional spacetime. It provides a universal expression for the Casimir energy which exhibits its dependence on the boundary conditions via the reflection amplitudes of the low energy particle excitations. We demonstrate the easy and straightforward applicability of the general expression by analyzing the free scalar field with Robin boundary condition and by rederiving the most important results available in the literature for this geometry.
\end{abstract}

\section{INTRODUCTION}

The Casimir force between two neutral macroscopic bodies in vacuum is considered as a manifestation of zero point fluctuations intrinsic to any quantum field theory (QFT). It has received a lot of interest recently both from the theoretical and from the experimental side (cf. the recent reviews [1] and the book by Milton [2]).

This force can be determined from the volume dependence of the ground state energy (Casimir energy). It is usually derived either by a summation of zero point fluctuations of the quantum field restricted to the domain in question, or by analyzing the statistical fluctuations of the interactions between the boundaries. Our new derivation is similar to the first approach in the sense that it uses the quantum fields living between the boundaries. However, we describe the quantum field theory in question in terms of its particle excitations rather than the field fluctuations. As a consequence we describe the Casimir energy as 'virtual particle' exchange between the two boundaries and our formula is expressed in terms of the analytically continued reflection amplitudes of these particles.

The interaction between the quantum field and the macroscopic bodies is most often described by imposing appropriate boundary conditions on the field. The aim of this paper is to explore and make explicit the dependence of the Casimir force on the boundary conditions obeyed by the field(s) in a geometry when the boundary conditions are given on two parallel planes. We achieve this by regarding the force as a physical manifestation of finite size effects in a boundary QFT (BQFT).

In QFT, finite size effects in a periodic box were considered by Lüscher [3], who showed how the correction to particle masses (infinite volume energies) can be expressed in terms of infinite volume scattering data $(S$ matrix elements). Following this general idea, we have recently determined the finite size correction to the ground state energy in 2 dimensional BQFT in terms of scattering data on the half line (reflection amplitudes). This explicitly describes how the ground state energy depends on the volume and it is obvious from the results in [4] that a generalization to BQFT in any spacetime dimen- sion is straightforward and leads to a description of the Casimir effect.

Boundary conditions in quantum field theory can be equally well described by a boundary state in a crossed channel. This formalism was worked out in detail for integrable $1+1$ dimensional BQFTs [5], but it can be extended to nonintegrable field theories in any number of spacetime dimensions. We show that this formalism allows an alternative method to derive the Casimir energy, and it has the advantage of using only renormalized and phenomenologically meaningful field theoretic quantities. A further advantage of using the boundary state formalism is that it provides a systematic (large volume) expansion for the Casimir energy in the interacting case.

Our main result is a large volume expression for the Casimir energy/force, valid in any BQFT, which depends only on the reflection amplitudes on the boundaries (although the boundary conditions considered allow transmission as well). These reflection amplitudes can be determined in a straightforward way in the half-infinite (one boundary) geometry, and using them in the expression for the Casimir energy makes it unnecessary to carry out the finite volume quantization separately in the various applications.

The paper is organized as follows: in Section II we introduce BQFTs and derive our main results for the volume dependence of their ground state energy using the boundary state formulation. Section III contains a description of several applications of the result in various BQFTs: First we derive a formula for the Casimir energy of a free massive boson subject to Robin type boundary condition. This expression is tested against the well-known Dirichlet, Neumann and massless limits. We further check our formula by recomputing the Casimir effect for parallel dielectric slabs separated by a vacuum slot and for a massless fermion subject to "bag boundary condition". We make our concluding remarks in Section IV. In the Appendix we confirm our result in a simple case by presenting the naive derivation based on mode summation tailor made for the framework of boundary QFTs. 


\section{DERIVATION OF THE MAIN FORMULA}

Here we summarize the main aspects of BQFTs and present the novel derivation of the Casimir energy. We follow the description of BQFTs along the line of 6$]$ extending the result of [5] for higher dimensions. In the process we show that this formalism makes it possible to establish a systematic (large volume) expansion of the Casimir energy even for interacting fields.

\section{A. Boundary state formalism}

To simplify the presentation consider a quantum field theory of a scalar field, $\Phi(t, x, \vec{y})$, in $D+1$ dimensions with Lagrangian

$$
\mathcal{L}=\frac{1}{2}\left(\partial_{t} \Phi\right)^{2}-\frac{1}{2}\left(\partial_{x} \Phi\right)^{2}-\frac{1}{2}(\vec{\partial} \Phi)^{2}-V(\Phi)
$$

where $\vec{y}$ is a $D-1$ dimensional position vector, $\vec{\partial}_{i}=$ $\frac{\partial}{\partial y_{i}}$. We also suppose that the spectrum consists of one particle type with mass $m$. The Hilbert space of the model can be spanned by asymptotic multi-particle states with momentum parameterized by $\left(k_{i}, \vec{k}_{i}\right)$ (where again $\vec{k}_{i}$ denotes a $D-1$ dimensional momentum vector):

$$
\mathcal{H}=\left\{\left|k_{1}, \vec{k}_{1} ; k_{2}, \vec{k}_{2} ; \ldots ; k_{n}, \vec{k}_{n}\right\rangle\right\}
$$

The energy of a one-particle state is

$$
\omega(k, \vec{k})=\sqrt{m^{2}+k^{2}+\vec{k}^{2}}
$$

In the asymptotic scattering configurations (large negative or positive time) the particles are distant from each other, and the spectrum can be described by free in or out particles so the energy of the multi-particle state is the sum of the individual energies. These two Hilbert spaces are connected by the scattering matrix

$$
\begin{aligned}
& S\left(\left\{k_{i}^{\prime}, \vec{k}_{i}^{\prime}\right\},\left\{k_{i}, \vec{k}_{i}\right\}\right)= \\
& \quad \text { out }\left\langle k_{1}^{\prime}, \vec{k}_{1}^{\prime} ; k_{2}^{\prime}, \vec{k}_{2}^{\prime} ; \ldots ; k_{n}^{\prime}, \vec{k}_{n}^{\prime} \mid k_{1}, \vec{k}_{1} ; k_{2}, \vec{k}_{2} ; \ldots ; k_{m}, \vec{k}_{m}\right\rangle^{\text {in }}
\end{aligned}
$$

which can be expressed in terms of the correlators

$$
\begin{aligned}
G\left(t_{1}, x_{1}, \vec{y}_{1} ; \ldots ; t_{N},\right. & \left.x_{N}, \vec{y}_{N}\right)= \\
& \left\langle 0\left|\Phi\left(t_{1}, x_{1}, \vec{y}_{1}\right) \ldots \Phi\left(t_{N}, x_{N}, \vec{y}_{N}\right)\right| 0\right\rangle
\end{aligned}
$$

via the LSZ reduction formula. The time evolution of the state is generated by the Hamiltonian

$H=\int_{-\infty}^{\infty} d x \int d \vec{y}\left(\frac{1}{2} \Pi^{2}+\frac{1}{2}\left(\partial_{x} \Phi\right)^{2}+\frac{1}{2}(\vec{\partial} \Phi)^{2}+V(\Phi)\right)$

where $\Pi=\partial_{t} \Phi$ is the conjugate momentum.

Let us suppose now that the theory is restricted to the half space $x<0$ and the boundary condition is given by specifying some boundary potential at $x=0$

$$
V_{B}(\Phi(0, \vec{y}, t))
$$

The essential observation is that we can have two different Hamiltonian descriptions of this system. We can take $t$ as the time variable and then the boundary is situated in space (i.e. it has a spacelike normal vector). In this case the Hilbert space consist of multi-particle states

$$
\mathcal{H}_{B}=\left\{\left|k_{\perp}, \vec{k}_{\|} ; k_{\perp}^{\prime},{\overrightarrow{k^{\prime}}}_{\|} ; \ldots\right\rangle_{B}\right\}
$$

where we indicate explicitly that the full momentum vector is composed of two components, one perpendicular and the other parallel to the boundary. The states are normalized in the following way:

$$
\begin{aligned}
& { }_{B}^{i n}\left\langle k_{\perp}^{\prime}, \vec{k}_{\|}^{\prime} \mid k_{\perp}, \vec{k}_{\|}\right\rangle_{B}^{i n}= \\
& (2 \pi)^{D} \omega\left(k_{\perp}, \vec{k}_{\|}\right) \delta\left(k_{\perp}-k_{\perp}^{\prime}\right) \delta^{(D-1)}\left(\vec{k}_{\|}-\vec{k}_{\|}^{\prime}\right)
\end{aligned}
$$

The subscript $B$ indicates these states satisfy the boundary condition. In the asymptotic past all the particles move towards the boundary: $k_{\perp}>0$. For $t \rightarrow-\infty$ they are separated far from each other and from the boundary forming the in state, which is a free state. For $t \rightarrow \infty$ all the scatterings and reflections are terminated they move backwards from the boundary are far from each other and from the boundary forming the free out state. These two Hilbert spaces are connected by the reflection matrix

$$
R_{\alpha \beta}={ }_{B}^{o u t}\langle\alpha \mid \beta\rangle_{B}^{\text {in }}
$$

The simplest case describes the one particle elastic reflection on the boundary and can be written as

$$
\begin{aligned}
& R\left(\theta, m_{\mathrm{eff}}\left(\vec{k}_{||}\right)\right)(2 \pi)^{D} \delta\left(\theta-\theta^{\prime}\right) \delta^{(D-1)}\left(\vec{k}_{\|}-\vec{k}_{\|}^{\prime}\right)= \\
& { }_{B}^{\text {out }}\left\langle k_{\perp}^{\prime}, \vec{k}_{\|}^{\prime} \mid k_{\perp}, \vec{k}_{\|}\right\rangle_{B}^{\text {in }}
\end{aligned}
$$

where we exploited the unbroken Poincaré symmetry (in the coordinates $t, \vec{y}$ ) and parameterized the perpendicular momentum as

$$
k_{\perp}=m_{\mathrm{eff}}\left(\vec{k}_{\|}\right) \sinh \theta \quad ; \quad m_{\mathrm{eff}}\left(\vec{k}_{\|}\right)=\sqrt{m^{2}+\vec{k}_{\|}^{2}}
$$

and then

$$
\delta\left(\theta-\theta^{\prime}\right)=m_{\mathrm{eff}}\left(\vec{k}_{\| \mid}\right) \cosh \theta \delta\left(k_{\perp}-k_{\perp}^{\prime}\right)
$$

The reflection factors can be expressed in terms of the correlators

$$
\begin{aligned}
& G\left(t_{1}, x_{1}, \vec{y}_{1} ; \ldots ; t_{N}, x_{N}, \vec{y}_{N}\right)= \\
& B\left\langle 0\left|\Phi\left(t_{1}, x_{1}, \vec{y}_{1}\right) \ldots \Phi\left(t_{N}, x_{N}, \vec{y}_{N}\right)\right| 0\right\rangle_{B}
\end{aligned}
$$

via the boundary reduction formula $[\underline{6}$. The time evolution of the state is generated by the Hamiltonian

$$
\begin{array}{r}
H_{B}=\int_{-\infty}^{0} d x \int d \vec{y}\left[\frac{1}{2} \Pi^{2}+\right. \\
+\frac{1}{2}\left(\partial_{x} \Phi\right)^{2}+\frac{1}{2}(\vec{\partial} \Phi)^{2} \\
\left.+V(\Phi)+\delta(x) V_{B}(\Phi)\right]
\end{array}
$$


In the second description of the boundary theory we can take $\tau=i x$ as time and $\xi=-i t$ as space variable in the Hamiltonian formalism [17]. The Hilbert space now is that of the bulk theory (without any boundary), and time evolution is given by the bulk Hamiltonian (this is the extension of the crossing symmetry of the bulk theory, and is analogous to the open-closed duality in string theory). In this case the boundary condition appears in time and serves as an initial state in calculating correlators:

$$
\begin{aligned}
& G\left(\tau_{1}, \xi_{1}, \vec{y}_{1} ; \ldots ; \tau_{N}, \xi_{N}, \vec{y}_{N}\right)= \\
& \left\langle 0\left|\Phi\left(\tau_{1}, \xi_{1}, \vec{y}_{1}\right) \ldots \Phi\left(\tau_{N}, \xi_{N}, \vec{y}_{N}\right)\right| B\right\rangle
\end{aligned}
$$

where now $|B\rangle$ is a state in the bulk Hilbert space $\mathcal{H}$. The boundary state $|B\rangle$ is in fact defined by the equality of correlation functions calculated in the two pictures. Using asymptotic completeness it can be expanded in the basis of the asymptotic in states of the bulk theory

$$
\begin{aligned}
|B\rangle= & \left\{1+K_{1} A_{i n}^{\dagger}(0,0)+\int_{0}^{\infty} \frac{d \theta}{2 \pi} \int \frac{d^{D-1} \vec{k}_{\|}}{(2 \pi)^{D-1}}\right. \\
& \left.K_{2}\left(\theta, m_{\mathrm{eff}}\left(\vec{k}_{||}\right)\right) A_{i n}^{\dagger}\left(-\theta,-\vec{k}_{||}\right) A_{i n}^{\dagger}\left(\theta, \vec{k}_{||}\right)+\ldots\right\}|0\rangle
\end{aligned}
$$

where $A^{\dagger}\left(\theta, \vec{k}_{\mid l}\right)$ is the in asymptotic creation operator and the ellipses denote the terms with higher particle number. Due to translational invariance in the spatial direction all the contributing states must have zero total momentum. Ghoshal and Zamolodchikov have shown 5] that the coefficient $K_{2}$ is related to the one-particle elastic reflection factor $R$ on the boundary by

$$
K_{2}\left(\theta, m_{\mathrm{eff}}\left(\vec{k}_{||}\right)\right)=R\left(\frac{i \pi}{2}-\theta, m_{\mathrm{eff}}\left(\vec{k}_{||}\right)\right)
$$

(here we simply treat $\vec{k}_{\|}$as a label for infinitely many species of two-dimensional particles of mass $m_{\mathrm{eff}}\left(\vec{k}_{||}\right)$, using the fact that $\vec{k}_{\|}$is conserved in the reflection off the boundary). The one-particle coefficient $K_{1}$ is only nonzero when the vacuum expectation value of the interpolating field $\Phi$ of the particle does not vanish. In this work we suppose that ${ }_{B}\langle 0|\Phi(t, x, \vec{y})| 0\rangle_{B}=0$ (which is the case for the usual applications). We plan to present the general case in a forthcoming publication, together with more detailed derivation of the form of the coefficient $K_{1}$ and $K_{2}$ using the boundary reduction formula obtained in [6], and a generalization to more complex particle spectra with several different masses. Here we restrict ourselves to a vanishing $K_{1}$ to keep the discussion simple. We remark that when the theory in the bulk is free and the reflection is elastic, the boundary state can be written in a closed form [18]

$$
\begin{aligned}
|B\rangle= & \exp \left(\int_{0}^{\infty} \frac{d \theta}{2 \pi} \int \frac{d^{D-1} \vec{k}_{||}}{(2 \pi)^{D-1}}\right. \\
& \left.K_{2}\left(\theta, m_{\mathrm{eff}}\left(\vec{k}_{||}\right)\right) A_{i n}^{\dagger}\left(-\theta,-\vec{k}_{||}\right) A_{i n}^{\dagger}\left(\theta, \vec{k}_{||}\right)\right)|0\rangle
\end{aligned}
$$

We note also that if we have more then one particle species, with the same mass $m$ created by $A_{i n}^{\dagger}\left(\theta, \vec{k}_{||}\right)_{j}$ (i.e. a multiplet), the formula for the boundary state changes as

$$
\begin{aligned}
& |B\rangle=\left\{1+\int_{0}^{\infty} \frac{d \theta}{2 \pi} \int \frac{d^{D-1} \vec{k}_{||}}{(2 \pi)^{D-1}}\right. \\
& \left.K_{2}^{i j}\left(\theta, m_{\mathrm{eff}}\left(\vec{k}_{||}\right)\right) A_{i n}^{\dagger}\left(-\theta,-\vec{k}_{||}\right)_{i} A_{i n}^{\dagger}\left(\theta, \vec{k}_{||}\right)_{j}+\ldots\right\}|0\rangle
\end{aligned}
$$

Boundary conditions considered in the context of the Casimir effect generally allow transmission as well, and such boundaries are called 'defects'. A suitable generalization of the above formalism can be obtained by a folding trick, which maps the defect into a boundary system 7]. Suppose now that a defect is located at $x_{0}$. In the crossed channel picture it can be represented by a defect operator which acts from the bulk Hilbert space of the $x<x_{0}$ system into that of the $x>x_{0}$ system. Lets denote the operator creating the particle for the $x<x_{0}$ domain as $A_{1}^{\dagger}$ while for the $x>x_{0}$ domain as $A_{2}^{\dagger}$. There are now four one-particle reflection amplitudes: $R^{ \pm}$are the ones preserving the species number 1,2 , while $T^{ \pm}$ are the ones changing 1 into 2 and 2 into 1 , respectively. Upon the folding correspondence, $R^{ \pm}$correspond to reflection processes on the two sides of the defect, while $T^{ \pm}$ describe the transmission amplitudes from one side to the other. Using the folding map to the boundary system we obtain the defect operator [7] as

$$
\begin{aligned}
D= & 1+\int_{\infty}^{\infty} \frac{d \theta}{4 \pi} \int \frac{d^{D-1} \vec{k}_{\|}}{(2 \pi)^{D-1}}( \\
& R^{+}\left(\frac{i \pi}{2}-\theta, m_{\mathrm{eff}}\left(\vec{k}_{||}\right)\right) A_{1}^{\dagger}\left(-\theta,-\vec{k}_{||}\right) A_{1}^{\dagger}\left(\theta, \vec{k}_{||}\right)+ \\
& T^{+}\left(\frac{i \pi}{2}-\theta, m_{\mathrm{eff}}\left(\vec{k}_{||}\right)\right) A_{1}^{\dagger}\left(-\theta,-\vec{k}_{||}\right) A_{2}\left(-\theta,-\vec{k}_{||}\right)+ \\
& T^{-}\left(\frac{i \pi}{2}-\theta, m_{\mathrm{eff}}\left(\vec{k}_{||}\right)\right) A_{1}\left(\theta, \vec{k}_{||}\right) A_{2}^{\dagger}\left(\theta, \vec{k}_{||}\right)+ \\
& \left.R^{-}\left(\frac{i \pi}{2}-\theta, m_{\mathrm{eff}}\left(\vec{k}_{||}\right)\right) A_{2}\left(\theta, \vec{k}_{||}\right) A_{2}\left(-\theta,-\vec{k}_{||}\right)\right)+\ldots
\end{aligned}
$$

which (with the same conditions as for the boundary state, but now elasticity is required for the combined oneparticle reflection/transmission amplitude [8] [19]) can be exponentiated to the form

$$
\begin{aligned}
D= & \exp \left\{\int_{\infty}^{\infty} \frac{d \theta}{4 \pi} \int \frac{d^{D-1} \vec{k}_{||}}{(2 \pi)^{D-1}}\right. \\
& \left(R^{+}\left(\frac{i \pi}{2}-\theta, m_{\mathrm{eff}}\left(\vec{k}_{||}\right)\right) A_{1}^{\dagger}\left(-\theta,-\vec{k}_{||}\right) A_{1}^{\dagger}\left(\theta, \vec{k}_{||}\right)+\right. \\
& T^{+}\left(\frac{i \pi}{2}-\theta, m_{\mathrm{eff}}\left(\vec{k}_{||}\right)\right) A_{1}^{\dagger}\left(-\theta,-\vec{k}_{||}\right) A_{2}\left(-\theta,-\vec{k}_{||}\right)+ \\
& T^{-}\left(\frac{i \pi}{2}-\theta, m_{\mathrm{eff}}\left(\vec{k}_{||}\right)\right) A_{1}\left(\theta, \vec{k}_{||}\right) A_{2}^{\dagger}\left(\theta, \vec{k}_{||}\right) \\
& \left.\left.+R^{-}\left(\frac{i \pi}{2}-\theta, m_{\mathrm{eff}}\left(\vec{k}_{||}\right)\right) A_{2}\left(\theta, \vec{k}_{||}\right) A_{2}\left(-\theta,-\vec{k}_{||}\right)\right)\right\}
\end{aligned}
$$




\section{B. Derivation of Casimir energy}

Let us now turn to the derivation of Casimir energy of a $D+1$ dimensional scalar field $\Phi(t, x, \vec{y})$ in a domain of width $L$ in $x$. To facilitate later applications, it is useful to consider the case when the field $\Phi(t, x, \vec{y})$ is allowed to penetrate through the ends of the domain in such a way, that in the two domains of width $R$ adjoining $L$, the dispersion relation of the asymptotic particles may be different from the vacuum one in $L$. The totally reflecting boundaries at $x= \pm(R+L / 2)$ are treated as auxiliary boundary conditions which are necessary in order to have a discrete spectrum; at the end of calculations we shall take the limit $R \rightarrow \infty$ and check that the results are independent of the choice of the auxiliary boundary conditions. So let us take the following situation in the coordinate $x$

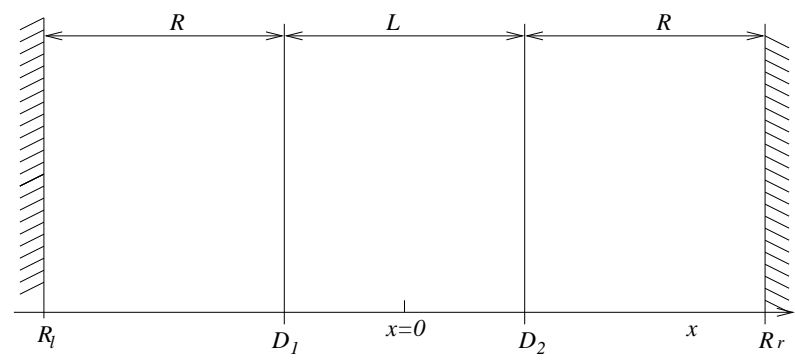

where total reflection occurs at $x_{l}=-R-L / 2$ and $x_{r}=R+L / 2$ with reflection factors $R_{l}\left(\theta, m_{\mathrm{eff}}\left(\vec{k}_{\| \mid}\right)\right)$and $R_{r}\left(\theta, m_{\text {eff }}\left(\vec{k}_{||}\right)\right)$and there are two "defects" (i.e. boundaries allowing transmission) located at $x_{1}=-L / 2$ and $x_{2}=L / 2$ with one-particle defect matrices

$$
D_{i}\left(\theta, m_{\mathrm{eff}}\left(\vec{k}_{||}\right)\right)=\left(\begin{array}{cc}
R_{i}^{+}\left(\theta, m_{\mathrm{eff}}\left(\vec{k}_{||}\right)\right) & T_{i}^{-}\left(\theta, m_{\mathrm{eff}}\left(\vec{k}_{||}\right)\right) \\
T_{i}^{+}\left(\theta, m_{\mathrm{eff}}\left(\vec{k}_{||}\right)\right) & R_{i}^{-}\left(\theta, m_{\mathrm{eff}}\left(\vec{k}_{||}\right)\right)
\end{array}\right)
$$

where $i=1,2$. The Hamiltonian of the system is denoted by $H_{B}$ and its Hilbert space by $\mathcal{H}_{B}$. It is the ground state energy of the system which is of interest for calculating the Casimir effect, i.e. the lowest eigenvalue of $H_{B}$, which can be evaluated via the partition function. Compactifying all infinite (temporal and spatial) dimensions on circles with perimeter $T$ we can calculate the partition function of our system in two different ways:

$$
\begin{aligned}
Z_{R}(L, T) & =\operatorname{Tr}_{\mathcal{H}_{B}} \mathrm{e}^{-T H_{B}} \\
& =\left\langle B_{l}\left|\mathrm{e}^{-R H_{x}^{(1)}} D_{1} \mathrm{e}^{-L H_{x}^{(2)}} D_{2} \mathrm{e}^{-R H_{x}^{(3)}}\right| B_{r}\right\rangle
\end{aligned}
$$

where $H_{x}^{(i)}$ is the periodic Hamiltonian in the $x$ channel in the three domains indexed by $i=1,2,3$. Inserting complete sets of bulk states, taking the limit $R \rightarrow \infty$ and normalizing the ground state energy in infinite space to $E_{0}=0$ we obtain

$$
\begin{aligned}
Z_{\infty}(L, T) & =\sum_{n}\left\langle B_{l} \mid 0\right\rangle\left\langle 0\left|D_{1}\right| n\right\rangle\left\langle n\left|D_{2}\right| 0\right\rangle\left\langle 0 \mid B_{r}\right\rangle \mathrm{e}^{-L E_{n}} \\
& =\sum_{n}\left\langle 0\left|D_{1}\right| n\right\rangle\left\langle n\left|D_{2}\right| 0\right\rangle \mathrm{e}^{-L E_{n}}
\end{aligned}
$$

where the dependence on the auxiliary boundary conditions $B_{l, r}$ drops out (since in the $R \rightarrow \infty$ limit only the vacuum state contributes from the expansions of $\left.\left|B_{l, r}\right\rangle\right)$. The first few terms can be written explicitly as

$$
\begin{array}{r}
1+\sum_{\theta, \vec{k}_{||}} \sum_{\theta^{\prime}, \vec{q}_{||}}\left\langle 0\left|D_{1}\right| \theta, \vec{k}_{||} ; \theta^{\prime}, \vec{q}_{||}\right\rangle\left\langle\theta, \vec{k}_{||} ; \theta^{\prime}, \vec{q}_{||}\left|D_{2}\right| 0\right\rangle \times \\
e^{-L\left(m_{\text {eff }}\left(\vec{k}_{||}\right) \cosh \theta+m_{\text {eff }}\left(\vec{q}_{||}\right) \cosh \theta^{\prime}\right)}+O\left(e^{-3 m L}\right)
\end{array}
$$

The term 1 is the contribution from the vacuum $(|n\rangle=$ $|0\rangle)$, the next term comes from two-particle terms in (3) and the higher-order corrections come from the higher multi-particle terms. This is a sort of cluster expansion similar to the one used in [4], valid for large values of the volume $L$. It is obvious from these expressions that the leading (two-particle) contribution depends only on $R_{1}^{-}$and $R_{2}^{+}$. The ground state (Casimir) energy (per unit transverse area) can be extracted from the partition function as

$$
E(L)=-\lim _{T \rightarrow \infty} \frac{1}{T^{D}} \log Z_{\infty}(L, T)
$$

The result is

$$
\begin{array}{r}
E(L)=-\int_{-\infty}^{\infty} \frac{d \theta}{4 \pi} \cosh \theta \int \frac{d^{D-1} \vec{k}_{\|}}{(2 \pi)^{D-1}} m_{\mathrm{eff}}\left(\vec{k}_{\|}\right) \\
R_{1}^{-}\left(\frac{i \pi}{2}+\theta, m_{\mathrm{eff}}\left(\vec{k}_{||}\right)\right) R_{2}^{+}\left(\frac{i \pi}{2}-\theta, m_{\mathrm{eff}}\left(\vec{k}_{\| \mid}\right)\right) \times \\
\mathrm{e}^{-2 m_{\mathrm{eff}}\left(\vec{k}_{||}\right) \cosh \theta L}+O\left(e^{-3 m L}\right)
\end{array}
$$

The correction terms correspond to higher particle terms in the expansion (3) of the defect operator $D$ and include the amplitudes of reflection/transmission processes involving more than one particle in at least one of the asymptotic states. These can be computed e.g. using a BQFT formulation as the one in [6], but it is obvious that they are suppressed by a factor $\mathrm{e}^{-m L}$ with respect to the leading order term due to the presence of at least one additional particle in the corresponding term of the expansion of the defect operator $D$.

The formula (5), describing Casimir effect to leading order at long distances, is the main result of this paper. Note that it is applicable in the presence of nontrivial bulk and boundary interactions: their effects at leading order are contained in the reflection factors $R^{ \pm}$, so as long as there is some theoretical or experimental input from which these can be determined the leading order contribution can be evaluated. In integrable (2D) boundary theories $R^{ \pm}$are obtained as solutions of the boundary Yang Baxter equation, and the bulk interaction manifests itself thorough the bulk S matrix appearing in this equation. In case of nonintegrable interacting bulk theories e.g. a perturbative expansion can be given to determine $R^{ \pm}$, (for more details see [6]).

Another important point is that this approach formulates the Casimir effect from an infrared viewpoint. Standard derivations of the Casimir effect (such as the one 
presented in the appendix) solve the microscopic field theory. This necessitates tackling diverse issues such as renormalization, and also the possibility that the infrared (long distance behaviour) may be quite different from the microscopic description of the theory (as is the case for example in QCD). Formula (15) is expressed in terms of the asymptotic particles, the long distance degrees of freedom, and provides a long distance expansion for Casimir energy. It describes a finite size effect in a boundary quantum field theory close in spirit to our previous investigation of the boundary Lüscher formula [4], and it is not difficult to see that the latter is just a special case of (5). As a consequence our formula has already been tested in interacting (integrable) 2D quantum field theories in [4]. (This is the only case to our knowledge where exact reflection factors of interacting BQFTs have been computed). Furthermore, the result (5) includes the contribution of states localized to the defects (called 'surface plasmons' in the literature), since they are taken into account as poles at imaginary rapidity of the reflection factors. This fact is also demonstrated explicitely using the zero mode summation method in Appendix A.

A very appealing property of the boundary state approach is its universality and we shall see in the next section that it indeed reproduces all the results previously known for the planar situation. But in those cases we can go even further, because in most calculations of the Casimir effect the bulk is free and the boundary scattering is elastic, therefore one can use the exponentiated boundary state (2) together with the analogous defect operator (4) which makes it possible to sum up all the multi-particle terms of the cluster expansion. The calculation to be done is essentially identical to the derivation of the boundary Thermodynamic Bethe Ansatz equation with trivial bulk $S$ matrix and reflection factors $R_{1}^{-}$and $R_{2}^{+}$, which was already performed in [9]. The only difference is that [9] supposes fermionic statistics and that in our case the reflection factors $R_{1}^{-}$and $R_{2}^{+}$are not unitary due to the existence of transmission, but these do not change the overall reasoning of the derivation. The result is

$$
\begin{gathered}
E(L)= \pm \int_{-\infty}^{\infty} \frac{d \theta}{4 \pi} \cosh \theta \int \frac{d^{D-1} \vec{k}_{\|}}{(2 \pi)^{D-1}} m_{\mathrm{eff}}\left(\vec{k}_{||}\right) \\
\log \left(1 \mp R_{1}^{-}\left(\frac{i \pi}{2}+\theta, m_{\mathrm{eff}}\left(\vec{k}_{||}\right)\right) R_{2}^{+}\left(\frac{i \pi}{2}-\theta, m_{\mathrm{eff}}\left(\vec{k}_{||}\right)\right)\right. \\
\mathrm{e}^{\left.-2 m_{\mathrm{eff}}\left(\vec{k}_{||}\right) \cosh \theta L\right)}
\end{gathered}
$$

(the upper/lower signs correspond to the bosonic/fermionic case, respectively). Although formula (5) is more general and adequate enough for the large distance regime, the theoretical situations considered in the literature can be directly compared to (6). It is obvious that for $L \gg m^{-1}$ (6) reduces to (5). The Casimir force is dominated by modes with momentum of the order $1 / L$; for large enough separation the bulk interaction can be dropped due to its short-ranged nature, and at the same time the characteristic energy is lower than the threshold for inelastic boundary scattering.

\section{APPLICATIONS}

To show the universal nature of these results we consider some applications. We demonstrate that taking the reflection factors from the literature and substituting into (6) immediately gives the Casimir energy without going through a detailed analysis of the microscopic quantum fluctuations.

As a first application we determine the Casimir energy for a massive free scalar field, $V(\Phi)=\frac{m^{2}}{2} \Phi^{2}$, subject to Robin boundary conditions, $V_{B}(\Phi)=\frac{c}{2} \Phi^{2}$, on two parallel hyperplanes. If the two planes are located at $x=0$ and $x=L$ the boundary conditions are given as

$$
\partial_{x} \Phi-\left.c_{1} \Phi\right|_{x=0}=0 ; \quad \partial_{x} \Phi+\left.c_{2} \Phi\right|_{x=L}=0 ; \quad c_{1}, c_{2} \geq 0,
$$

and the reflection amplitudes on these planes can be written as

$$
R_{j}\left(\theta, m_{\mathrm{eff}}\left(\vec{k}_{||}\right)\right)=\frac{m_{\mathrm{eff}}\left(\vec{k}_{||}\right) \sinh \theta-i c_{j}}{m_{\mathrm{eff}}\left(\vec{k}_{||}\right) \sinh \theta+i c_{j}} ; \quad j=1,2 .
$$

Note that they have the same form as in the two dimensional case, but now they depend also on $\vec{k}_{\| \mid}$via the rapidity parameterization $k_{\perp}=m_{\mathrm{eff}}\left(\vec{k}_{||}\right) \sinh \theta$. When $\theta$ is continued to $\theta \rightarrow \theta+i \frac{\pi}{2}$, we have

$$
R_{j}\left(\theta+i \frac{\pi}{2}, m_{\mathrm{eff}}\left(\vec{k}_{||}\right)\right)=\frac{m_{\mathrm{eff}}\left(\vec{k}_{||}\right) \cosh \theta-c_{j}}{m_{\mathrm{eff}}\left(\vec{k}_{||}\right) \cosh \theta+c_{j}} ; \quad j=1,2 .
$$

Introducing the variable $q$ by $m_{\mathrm{eff}}\left(\vec{k}_{||}\right) \cosh \theta=$ $\sqrt{m^{2}+q^{2}}$ and performing the angular integrations one obtains

$$
\begin{aligned}
& E(L)=\frac{1}{(4 \pi)^{D / 2} \Gamma(D / 2)} \int_{0}^{\infty} d q q^{D-1} \\
& \log \left(1-\frac{\sqrt{m^{2}+q^{2}}-c_{1}}{\sqrt{m^{2}+q^{2}}+c_{1}} \frac{\sqrt{m^{2}+q^{2}}-c_{2}}{\sqrt{m^{2}+q^{2}}+c_{2}} e^{-2 L \sqrt{m^{2}+q^{2}}}\right) .
\end{aligned}
$$

This formula is a new result of the present paper. To show its correctness we consider two already known limiting cases. First consider the limit when both $c_{j} \rightarrow 0$ or $c_{j} \rightarrow \infty$ corresponding to Neumann or Dirichlet boundary conditions for $\Phi$. In both cases the coefficient of the exponent in the logarithm becomes one, and we obtain the Ambjorn-Wolfram result for Dirichlet boundary conditions [10] as reported in Milton's book [2]. In the second limit we let the mass of the scalar field vanish $m \rightarrow 0 ;$ then

$$
\begin{aligned}
& E(L)= \\
& \frac{1}{(4 \pi)^{D / 2} \Gamma(D / 2)} \int_{0}^{\infty} d q q^{D-1} \log \left(1-\frac{q-c_{1}}{q+c_{1}} \frac{q-c_{2}}{q+c_{2}} e^{-2 L q}\right) .
\end{aligned}
$$


It is straightforward to show that this result coincides with that obtained in 11] for the Casimir energy per unit area of a massless scalar field with Robin boundary conditions.

The second application concerns the Casimir force between parallel dielectric slabs separated by a vacuum slot of width $L$, a problem first investigated by Lifshitz and collaborators almost fifty years ago [12]. In this geometry the dielectric constants in the three regions are:

$$
\begin{gathered}
\epsilon(x)=\epsilon_{1} ; \quad x<0 ; \quad \epsilon(x)=\epsilon_{2} ; \quad L<x ; \\
\epsilon(x)=1 ; \quad 0<x<L .
\end{gathered}
$$

In this case $R_{1,2}^{ \pm}\left(i \frac{\pi}{2}+\theta\right)$ are nothing else but the (appropriate analytic continuations of the) ordinary reflection amplitudes of electromagnetic waves incident from vacuum at the plane interface between the vacuum and the dielectric materials, given in many textbooks (e.g. [13]). Indeed denoting again by $\left(k_{\perp}, \overrightarrow{k_{\|}}\right), \omega$ the wave vector and frequency of the electromagnetic radiation $\left(\vec{k}_{||}\right.$being the component parallel, while $k_{\perp}$ the one perpendicular to the plane interface) the reflection amplitudes are

$$
R_{\mathrm{perp}}^{(i)}\left(\omega, \vec{k}_{\|}\right)=\frac{\sqrt{\omega^{2}-\vec{k}_{\|}^{2}}-\sqrt{\epsilon_{i} \omega^{2}-\vec{k}_{\|}^{2}}}{\sqrt{\omega^{2}-\vec{k}_{\|}^{2}}+\sqrt{\epsilon_{i} \omega^{2}-\vec{k}_{\|}^{2}}}, \quad \epsilon_{i}=\epsilon_{i}(\omega),
$$

when the electric field $\vec{E}$ is perpendicular to the plane of incidence, and

$$
R_{\mathrm{par}}^{(i)}\left(\omega, \vec{k}_{\|}\right)=\frac{\epsilon_{i} \sqrt{\omega^{2}-\vec{k}_{\|}^{2}}-\sqrt{\epsilon_{i} \omega^{2}-\vec{k}_{\|}^{2}}}{\epsilon_{i} \sqrt{\omega^{2}-\vec{k}_{\|}^{2}}+\sqrt{\epsilon_{i} \omega^{2}-\vec{k}_{\|}^{2}}}, \quad \epsilon_{i}=\epsilon_{i}(\omega),
$$

when $\vec{E}$ is parallel to the plane of incidence and $i=1,2$. (To obtain these expressions we assumed that the permeabilities of the slabs are unity, $\mu_{i}=1$ ). Continuing $\theta \rightarrow \theta+i \frac{\pi}{2}$ corresponds to continuing $\omega$ to purely imaginary values $\omega \rightarrow m_{\mathrm{eff}}\left(\vec{k}_{\|}\right) i \sinh \theta=i \zeta$, and the reflection amplitudes become $\left(q^{2}=\vec{k}_{\|}^{2}\right)$ :

$$
\begin{aligned}
R_{\mathrm{perp}}^{(i)}(i \zeta, q) & =\frac{\sqrt{\zeta^{2}+q^{2}}-\sqrt{\epsilon_{i} \zeta^{2}+q^{2}}}{\sqrt{\zeta^{2}+q^{2}}+\sqrt{\epsilon_{i} \zeta^{2}+q^{2}}} \\
R_{\mathrm{par}}^{(i)}(i \zeta, q) & =\frac{\epsilon_{i} \sqrt{\zeta^{2}+q^{2}}-\sqrt{\epsilon_{i} \zeta^{2}+q^{2}}}{\epsilon_{i} \sqrt{\zeta^{2}+q^{2}}+\sqrt{\epsilon_{i} \zeta^{2}+q^{2}}} .
\end{aligned}
$$

Since the electromagnetic field can have both polarizations, one obtains the following form for the Casimir energy per unit area

$$
\begin{aligned}
E(L)= & \frac{1}{8 \pi^{2}} \int_{0}^{\infty} d q^{2} \int_{0}^{\infty} d \zeta[ \\
& \log \left(1-R_{\mathrm{perp}}^{(1)}(i \zeta, q) R_{\mathrm{perp}}^{(2)}(i \zeta, q) \mathrm{e}^{-2 L \sqrt{q^{2}+\zeta^{2}}}\right)+ \\
& \left.\log \left(1-R_{\mathrm{par}}^{(1)}(i \zeta, q) R_{\mathrm{par}}^{(2)}(i \zeta, q) \mathrm{e}^{-2 L \sqrt{q^{2}+\zeta^{2}}}\right)\right]
\end{aligned}
$$

The Casimir force $\mathcal{F}(L)=-\partial E(L) / \partial L$ computed from this expression agrees with that of Lifshitz et al. as reported in the reviews [1, 2].

Next we consider the Casimir energy of a massless fermion field in $1+3$ dimensions subject to the "bag boundary condition" in our planar geometry:

$$
\left.\left(1-i \gamma^{3}\right) \psi\right|_{x=0}=0 \quad,\left.\quad\left(1+i \gamma^{3}\right) \psi\right|_{x=L}=0 \quad .
$$

Using the chiral representation of Dirac matrices one readily shows that these boundary conditions break chirality but commute with $\Sigma_{3}=\left(\begin{array}{cc}\sigma_{3} & 0 \\ 0 & \sigma_{3}\end{array}\right)$. Furthermore they imply that for both the positive $(u)$ and the negative $(v)$ eigenvectors of $\Sigma_{3}$ the reflection amplitudes are constants, satisfying $R_{1(s)}^{-}(i \pi / 2+\theta) R_{2(s)}^{+}(i \pi / 2+\theta)=-1$ for $s=u, v$. Thus (taking into account crossing-unitarity (A8)

$$
E(L)=-2 \frac{1}{4 \pi^{2}} \int_{0}^{\infty} d q q^{2} \log \left(1+e^{-2 L q}\right),
$$

which indeed coincides with the known result [2, 14].

\section{CONCLUDING REMARKS}

The main result of the paper is formula (5) which expresses the Casimir energy in large volume in a general form valid for any QFT - even for theories interacting in the bulk - in terms of the reflection amplitudes (infrared data). It passed the test of interacting but integrable two dimensional quantum field theories in [4]. The boundary state approach provides a systematic infrared (large volume) expansion, which in the case of noninteracting bulk and elastic boundary scattering can be summed up leading to ([6). In order to check the result for noninteracting bulk we present in the appendix an alternative derivation using the naive mode summation method.

In the usual calculations of the Casimir effect corrections for temperature and roughness effects must be made before comparing to the experiment. Both corrections can be introduced into (56) in a straightforward manner, following the usual procedure in the literature (cf. [2]) see also [16]. In the case of a massive theory with mass gap $m$ the terms coming from higher particle corrections to (11) and (3) are suppressed by $\exp (-m L)$. For theories with a zero mass gap the situation is different and indeed the asymptotic state formalism is not wellfounded if interaction with massless particles (such as photons) is taken into account, due to infrared divergencies. In the case of electromagnetic field the corrections to (6) are known to be suppressed by $\alpha \lambda / L$ where $\lambda$ is the Compton wave length of the electron and $\alpha$ is the fine structure constant (Chapter 13 of [2]), due to the fact that the self-interaction of the electromagnetic field arises only through radiative corrections and the leading one is an electron loop. 
The striking fact about the general formulae (56) is that they are manifestly finite, and only depend on physical quantities, such as the dispersion relation of asymptotic particles and their reflection amplitudes on the boundaries. This is even more emphasized in the boundary state formalism, where the bulk and boundary divergences appearing in usual derivations of the Casimir effect are manifestly absent, and the whole derivation is performed in terms of finite (renormalized) physical objects. The appearance of divergences in the naive method of summing zero-point energies is a general feature when one calculates the interaction energy of point-like sources by integrating the energy stored in the field generated by them. The divergences are then eliminated by noticing that they contribute to the self-energy of the sources themselves and are present even at infinite separation. These divergences are absorbed by renormalization, but they are entirely unphysical and eliminated when expressing the measurable energy differences in terms of physically meaningful, finite quantities. Further bonus of the general formulae (56) is that the reflection factors appearing in them can be calculated easily in the semiinfinite geometry, e.g. using perturbation theory along the lines of [6].

\section{Acknowledgments}

This research was partially supported by the EC network "EUCLID", contract number HPRN-CT-200200325, and Hungarian research funds OTKA D42209, T037674, T043582 and TS044839. GT was also supported by a Széchenyi István Fellowship.

\section{Appendix A: CASIMIR ENERGY BY MODE SUMMATION}

Here we want to support our derivation coming from the boundary state formalism by obtaining the analogous result using a new version of the mode summation method for fluctuating fields which are free in the bulk. Ideas somewhat similar to these have been used in a similar framework earlier in [15] and their findings also support our general expression. The novel viewpoints of our approach are twofold: first we concentrate only on the finite size piece of the ground state energy that should be independent of regularization and renormalization thus we do not need to discuss the regularization/renormalization of the (infinite space) bulk/boundary ground state energies, since eventually these are subtracted. The second feature of our approach is that we do not solve the quantization condition for the zero point frequencies explicitly but use only the functional form of these equations to obtain a general expression for the ground state energy containing only the reflection amplitudes.

For simplicity let us start with the $1+1$ dimensional free scalar field $\Phi(t, x)$ of mass $m$ in a domain described earlier. We assume that in all three domains the frequency of the modes of $\Phi$ is the same, but the wave vectors $k^{\prime}, k^{\prime \prime}$ may be different from $k$ (e.g. the mass of the scalar field is different in the three domains), but they can be regarded as functions of $k$. All the wave number parameters $k, k^{\prime}, k^{\prime \prime}$ are chosen positive. Then for the plane wave modes one has the situation displayed in the following figure

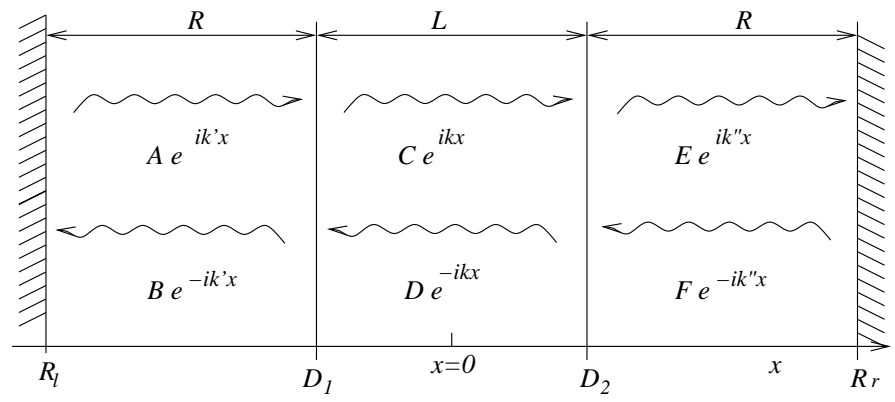

where total reflection occurs at $x_{l}=-R-L / 2$ and $x_{r}=R+L / 2$ with reflection factors $R_{l}$ and $R_{r}$

$$
\begin{aligned}
A e^{-i k^{\prime}\left(R+\frac{L}{2}\right)} & =B e^{i k^{\prime}\left(R+\frac{L}{2}\right)} R_{l}\left(k^{\prime}\right) \\
F e^{-i k^{\prime \prime}\left(R+\frac{L}{2}\right)} & =E e^{i k^{\prime \prime}\left(R+\frac{L}{2}\right)} R_{r}\left(k^{\prime \prime}\right),
\end{aligned}
$$

and the two "defects" (i.e. boundaries allowing transmission) located at $x_{1}=-L / 2$ and $x_{2}=L / 2$ describe one-particle scattering with defect matrices

$$
D_{i}(k)=\left(\begin{array}{cc}
R_{i}^{+}(k) & T_{i}^{-}(k) \\
T_{i}^{+}(k) & R_{i}^{-}(k)
\end{array}\right), \quad i=1,2
$$

In the case of elastic defect scattering their is no particle production and the defect matrices are unitary. They connect the incoming and outgoing amplitudes as follows

$$
\begin{aligned}
\left(\begin{array}{c}
B \mathrm{e}^{+i k^{\prime} L / 2} \\
C \mathrm{e}^{-i k L / 2}
\end{array}\right) & =D_{1}(k)\left(\begin{array}{c}
A \mathrm{e}^{-i k^{\prime} L / 2} \\
D \mathrm{e}^{+i k L / 2}
\end{array}\right) \\
\left(\begin{array}{c}
D \mathrm{e}^{-i k L / 2} \\
E \mathrm{e}^{+i k^{\prime \prime} L / 2}
\end{array}\right) & =D_{2}(k)\left(\begin{array}{c}
C \mathrm{e}^{+i k L / 2} \\
F \mathrm{e}^{-i k^{\prime \prime} L / 2}
\end{array}\right)
\end{aligned}
$$

Unitarity and time-reversal gives the relations

$$
\begin{array}{cll}
D_{i}(k)^{\dagger} D_{i}(k)=1 & D_{i}(k)^{-1}=D_{i}(-k) \\
R_{r, l}(k)^{*} R_{r, l}(k)=1 & , & R_{r, l}(k)^{-1}=R_{r, l}(-k)
\end{array}
$$

Consistency of the homogenous linear system (A1 A2 is expressed by the quantization condition

$$
Q\left(k_{n}\right)=0
$$

where

$$
\begin{array}{r}
Q(k)=\left(1-R_{l}(k) R_{1}^{+}(k) e^{i 2 k^{\prime} R}\right)\left(1-R_{r}(k) R_{2}^{-}(k) e^{i 2 k^{\prime \prime} R}\right)- \\
e^{i 2 k L} R_{1}^{-}(k) R_{2}^{+}(k)\left(1-\frac{R_{l}(k) e^{i 2 k^{\prime} R}}{R_{1}^{+}(-k)}\right)\left(1-\frac{R_{r}(k) e^{i 2 k^{\prime \prime} R}}{R_{2}^{-}(-k)}\right)
\end{array}
$$


and we exploited (A3) to write

$$
\operatorname{det} D_{i}=R_{i}^{-}(k) / R_{i}^{+}(-k)=R_{i}^{+}(k) / R_{i}^{-}(-k)
$$

Due to unitarity the quantization condition can only have real and purely imaginary solutions. For each real solution $k_{n},-k_{n}$ is also a solution, and $k_{0}=0$ always solves (A4). The purely imaginary solutions located at $k_{j}=i \kappa_{j}$ $\left(0<\kappa_{j}<m\right)$ are related to the poles of the reflection and transmissions factors and correspond to defect and boundary bound states. Then the ground state energy of the system is given by the sum of zero modes, which can be written in an integral form

$$
\begin{aligned}
& E(L, R)=\sum_{\left\{k_{n}>0\right\}} \frac{1}{2} \omega\left(k_{n}\right)+\sum_{j} \frac{1}{2} \omega\left(i \kappa_{j}\right)= \\
& \frac{1}{2}\left\{\frac{1}{2}\left(\int_{C_{+}}+\int_{C_{-}}-\int_{C_{0}}\right)+\sum_{j} \oint_{C_{j}}\right\} \frac{d k}{2 \pi i} \frac{Q^{\prime}(k)}{Q(k)} \omega(k)
\end{aligned}
$$

where $Q^{\prime}(k)=\frac{d Q}{d k}, \omega(k)=\sqrt{k^{2}+m^{2}}, \hbar$ is set to one and the contours are shown on the figure

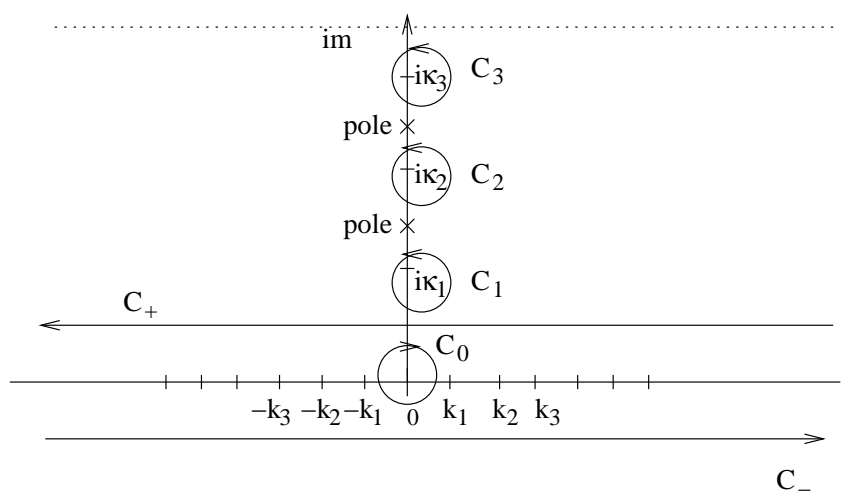

The poles on the figure denote the poles of the $Q$ function originating from the poles of the reflection and transmission amplitudes. Contrary to $\kappa_{j}$, which do depend on the volume, the location of these poles is independent of $L, R$.

We concentrate on the $L$ dependence so the $L$ independent $C_{0}$ integral can be dropped. Next we analyze the contribution of $C_{-}$. Using (A3) we can relate

$$
\begin{aligned}
\frac{Q^{\prime}(-k)}{Q(-k)} & -\frac{Q^{\prime}(k)}{Q(k)}=-2 i L-2 i R\left(\frac{d k^{\prime}}{d k}+\frac{d k^{\prime \prime}}{d k}\right) \\
& -\frac{d}{d k} \log R_{r}-\frac{d}{d k} \log \left(\operatorname{det} D_{1} D_{2}\right)-\frac{d}{d k} \log R_{l} .
\end{aligned}
$$

The first two terms on the right hand side (proportional to the volumes $L, R$ ) correspond to divergent bulk energy contributions. The other terms here correspond to infinite boundary and defect energies which are independent of the volumes $(L, R)$. The precise definition of these terms needs some regularization and renormalization, but they give no contribution to the Casimir force since they are present in the infinite system as well.
Therefore they can be dropped and then the $C_{+}$and $C_{-}$ integral terms give identical results.

Thus the relevant contribution to the Casimir energy can be written in terms of the variable $m \sinh \theta=k$ as

$$
E(L, R)=-m \int_{-\infty+i \eta}^{\infty+i \eta} \frac{d \theta}{4 \pi i} \cosh \theta \frac{Q^{\prime}(\theta)}{Q(\theta)}+\sum_{j} \frac{1}{2} \omega\left(i \kappa_{j}\right) .
$$

(where $\eta$ is a small positive parameter). Next we shift the contour to $\eta=\frac{\pi}{2}$ which eliminates the discrete sum via the contributions from the purely imaginary zeros of $Q(\theta)$. There can be additional contributions from poles of $Q(\theta)$ on the imaginary axis, but their positions are independent of the volume and therefore they only contribute volume independent terms which can be dropped. Integrating by parts and introducing the new variable $\theta \rightarrow \frac{i \pi}{2}+\theta$ we obtain

$$
E(L, R)=m \int_{-\infty}^{\infty} \frac{d \theta}{4 \pi} \cosh \theta \log Q\left(\frac{i \pi}{2}+\theta\right) .
$$

In the limit $R \rightarrow \infty$

$$
\begin{aligned}
& E(L)=m \int_{-\infty}^{\infty} \frac{d \theta}{4 \pi} \cosh \theta \\
& \quad \log \left(1-R_{1}^{-}\left(\frac{i \pi}{2}+\theta\right) R_{2}^{+}\left(\frac{i \pi}{2}+\theta\right) \mathrm{e}^{-2 m \cosh \theta L}\right) .
\end{aligned}
$$

It is straightforward to generalize these expressions to the $D+1$ dimensional case when the boundary conditions are specified on two parallel $D-1$ dimensional hyperplanes. Introducing the component parallel $\left(\vec{k}_{||}\right)$respectively perpendicular $\left(k_{\perp}\right)$ to the hyperplanes one can write

$$
\begin{aligned}
\vec{k}= & \left(k_{\perp}, \vec{k}_{\|}\right) ; \quad \omega^{2}-k_{\perp}^{2}=m^{2}+\vec{k}_{\|}^{2} \equiv m_{\mathrm{eff}}^{2}\left(\vec{k}_{\|}\right) ; \\
& k_{\perp}=m_{\mathrm{eff}}\left(\vec{k}_{\|}\right) \sinh \theta .
\end{aligned}
$$

We assume that the system is translationally invariant in the direction of the hyperplanes, and so the reflection and transmission processes preserve $\vec{k}_{\|}$. Therefore we can apply the previous considerations to the decoupled one-dimensional systems labeled by the parameter $\vec{k}_{||}$and sum up their contributions. Note that both $\omega$ and the reflection/transmission amplitudes depend on $\vec{k}_{\| \mid}$, but we shall not write this out explicitely. The Casimir energy per unit transverse area can be written as

$$
\begin{aligned}
& E(L, R)= \\
& \int \frac{d^{D-1} \vec{k}_{\| \mid}}{(2 \pi)^{D-1}} m_{\mathrm{eff}}\left(\vec{k}_{\| \mid}\right) \int_{-\infty}^{\infty} \frac{d \theta}{4 \pi} \cosh \theta \log Q\left(\frac{i \pi}{2}+\theta\right) .
\end{aligned}
$$

From this, the Casimir energy between the two defects can be obtained by taking the limit $R \rightarrow \infty$

$$
\begin{aligned}
& E(L)=\int \frac{d^{D-1} \vec{k}_{\|}}{(2 \pi)^{D-1}} m_{\mathrm{eff}}\left(\vec{k}_{||}\right) \int_{-\infty}^{\infty} \frac{d \theta}{4 \pi} \cosh \theta \\
& \log \left(1-R_{1}^{-}\left(\frac{i \pi}{2}+\theta\right) R_{2}^{+}\left(\frac{i \pi}{2}+\theta\right) \mathrm{e}^{-2 m_{\mathrm{eff}}\left(\vec{k}_{||}\right) \cosh \theta L}\right) .
\end{aligned}
$$


Note that the latter result is independent of the auxiliary boundary conditions $R_{r}$ and $R_{l}$ which can be expected on physical grounds. Taking into account the crossingunitarity property satisfied by the reflection amplitudes [5, 7] which for free bosons/fermions takes the form

$$
R\left(\frac{i \pi}{2}+\theta\right)= \pm R\left(\frac{i \pi}{2}-\theta\right)
$$

one can see that this result is in a complete agreement with the formula obtained form the boundary state formalism previously (6). (The results in (A6] A7) should be multiplied by $(-1)$ in case of computing the Casimir energy of fermionic fields, since their vacuum energy is $\left.-\frac{1}{2} \sum \omega(k)\right)$.

If there are more than one fields in the problem, or the fields have more than one component one has to sum up the contribution of all the fields (components) to obtain the Casimir energy.
[1] K.A. Milton: The Casimir effect: recent controversies and progress, J. Phys. A37 (2004) R209, hep-th/0406024

M. Bordag, U. Mohideen and V.M. Mostepanenko: New developments in the Casimir effect, Phys. Rep. 353 (2001) 1-205, quant-ph/0106045

[2] K.A. Milton: The Casimir Effect: Physical Manifestations of Zero-Point Energy. Published by World Scientific, 2001.

[3] M. Lüscher: Volume dependence of the energy spectrum in massive quantum field theories. 1. Stable particle states, Comm. Math. Phys. 104 (1986) 177.

M. Lüscher: Volume dependence of the energy spectrum in massive quantum field theories. 2 . Scattering states, Comm. Math. Phys. 105 (1986) 153-188.

[4] Z. Bajnok, L. Palla and G. Takács: Finite size effects in quantum field theories with boundary from scattering data, Nucl. Phys. B716 (2005) 519-542, hep-th/0412192

[5] S. Ghoshal and A.B. Zamolodchikov: Boundary S matrix and boundary state in two-dimensional integrable quantum field theory, Int. J. Mod. Phys. A9 (1994) 3841-3886 (Erratum-ibid. A9 4353), hep-th/9306002

[6] Z. Bajnok, G. Böhm and G. Takács: Boundary reduction formula, J. Phys. A35 (2002) 9333-9342, hep-th/0207079

Z. Bajnok, G. Böhm and G. Takács: On perturbative quantum field theory with boundary, Nucl. Phys. B682 (2004) 585-617, hep-th/0309119

[7] Z. Bajnok and A. George: From defects to boundaries, hep-th/0404199 Int. J. Mod. Phys. A, in press.

[8] G. Delfino, G. Mussardo and P. Simonetti: Statistical models with a line of defect, Phys. Lett. B328 (1994) 123-129, hep-th/9403049

[9] A. Leclair, G. Mussardo, H. Saleur and S. Skorik: Boundary energy and boundary states in integrable quantum field theories, Nucl. Phys. B453 (1995) 581-618, hep-th/9503227
[10] J. Ambjorn and S. Wolfram: Properties of the vacuum. 2. Electrodynamic, Ann. Phys. 147 (1983) 33.

[11] L. C. de Albuquerque, R. M. Cavalcanti: Casimir effect for the scalar field under Robin boundary conditions: A functional integral approach, $J$. Phys. A37 (2004) 7039-7050, hep-th/0311052

A. Romeo and A. Saharian: Casimir effect for scalar fields under Robin boundary conditions on plates, J. Phys. A: Math Gen. 35 (2002) 1297-1320.

[12] E.M. Lifshitz, Zh. Eksp. Teor. Fiz. 29 (1956) 94 [Soviet Phys. JETP 2 (1956) 73].

I.D. Dzyaloshinskii, E.M. Lifshitz and L.P. Pitaevskii, Usp. Fiz. Nauk 73 (1961) 381 [Soviet Phys. Usp. 4 (1961) 153].

L.D. Landau and E.M. Lifshitz: Electrodynamics of Continous Media.Pergamon, Oxford, 1960.

[13] J.D. Jackson: Classical Electrodynamics. Published by John Wiley \& Sons, Inc., 1998 (3rd edition).

[14] K. Johnson: The M.I.T. Bag Model, Acta Phys. Pol. B6 (1975) 865.

[15] M.T. Jaekel and S. Reynaud: Casimir force between partially transmitting mirrors, Journal de Physique I1 (10): (1991) 1395-1409, quant-ph/0101067

[16] Paulo A. Maia Neto, A. Lambrecht and S. Reynaud: Casimir effect with rough metallic mirrors, Phys. Rev. A72 (2005) 012115, quant-ph/0505086

[17] In the Euclidean formalism this corresponds to swapping Euclidean time with the space variable $x$, which is manifestly a symmetry of the theory in the bulk.

[18] In 1+1 dimensions this can be extended to any integrable QFT with integrable boundary condition 5].

[19] We remark that integrable defects (which are exactly the ones for which the defect operator can be exponentiated) with nontrivial reflection and transmission at the same time are only possible when the bulk scattering is trivial 8]. This only means a restriction in $1+1$ dimensions, where integrable theories with nontrivial bulk scattering matrices are possible. In higher space-time dimension the conditions for the exponential form of the defect operator include the triviality of the bulk $S$ matrix anyway. 